\title{
Erros do Canal de Retorno em Sistemas que Empregam Modulação Adaptativa
}

\author{
Ingrid de Freitas Castro Machado e Juraci Ferreira Galdino
}

\begin{abstract}
Resumo-Este trabalho trata da avaliação de desempenho de sistemas de comunicação que empregam modulação adaptativa e cujos canais avante e de retorno são caracterizados pelo efeito de desvanecimento plano. Atenção especial é dedicada à análise e ao combate aos efeitos provocados pelos erros no canal de retorno. Tais erros podem causar o surgimento de uma região de inviabilidade na qual o sistema de modulação adaptativa não consegue atender aos requisitos de probabilidade de erro de bit máxima. Neste trabalho avalia-se o desempenho de duas técnicas propostas na literatura para combater esses distúrbios, porém aqui se adota uma modelagem do canal de retorno diferente da considerada naqueles trabalhos, e que permite estabelecer cenários nos quais o emprego dessas técnicas propicia bons resultados, bem como permite relacionar os valores dos parâmetros nelas adotadas com os dos tipicamente encontrados em um sistema de comunicação em canais com desvanecimento. Uma segunda contribuição deste trabalho é considerar na avaliação de desempenho dessas técnicas o erro de estimação do canal de retorno.
\end{abstract}

Palavras-Chave-Modulação adaptativa, avaliação de desempenho, desvanecimento plano, canal de retorno.

Abstract-This work addresses the performance evaluation of adaptive modulation techniques in communication systems whose forward and feedback channels are characterized by the flat fading. A special attention is dedicated to the analysis and solution of the effects caused by errors at the feedback channel. Such errors can cause the appearance of an outage region in which the adaptive modulation system cannot attend the requisites of maximum bit error probability. The work evaluates the performance of two techniques proposed in the literature to mitigate these disturbances. In this paper, however, it was chosen a more complex model of the feedback channel, but which permits to establish scenarios where the implementation of these techniques provide good results. It also permits to relate the adopted parameters values with the ones typically found in a channel communication system with fading. A second contribution of this work is take into account, in the performance evaluation of these techniques, the estimative error of the feedback channel.

Keywords-Adaptive modulation, performance evaluation, flat fading, feedback channel.

\section{INTRODUÇÃO}

Em contraposição às técnicas convencionais [1], as modulações adaptativas permitem variar a constelação durante a transmissão da informação, possibilitando, dessa maneira, adaptar as formas de onda na saída do transmissor de acordo com as condições de propagação do canal de comunicação [2]. Quando o canal apresenta excelentes condições de propagação é possível atingir reduzidas taxas de erro usando modulações com elevada Eficiência Espectral (EE). Por outro lado, quando

Ingrid de Freitas Castro Machado e Juraci Ferreira Galdino, Departamento de Engenharia Elétrica, Instituto Militar de Engenharia, Rio de Janeiro, Brasil, E-mails: ingridcastrorj@yahoo.com.bre galdino@ime.eb.br. o canal apresenta severas condições de propagação, para se obter baixos valores de taxa de erro de bit (BER - do inglês Bit Error Rate) deve-se adotar uma modulação com poucos pontos na constelação, e que, portanto, possui baixa eficiência espectral.

Em canais caracterizados pelo efeito de desvanecimento plano, cenário de comunicação de interesse neste trabalho, as condições de propagação podem ser avaliadas por meio da Razão Sinal Ruído (RSR) instantânea $(\gamma)$. Nesses casos, quanto maior a RSR melhores as condições de propagação do canal.

Para obter a adaptação, os receptores de sistemas que empregam modulação adaptativa estimam a RSR instantânea, comparam essa estimativa com limiares de $\operatorname{RSR}\left(\lambda_{i}\right)$ para definir a modulação mais indicada para as atuais condições de propagação do canal. Essa informação é transmitida por meio de um canal reverso ou de retorno ao transmissor, usando geralmente uma modulação fixa, onde será empregada na escolha da modulação a ser adotada na transmissão do próximo bloco de dados.

Os limiares mencionados previamente são obtidos pela resolução de um problema de otimização com restrição, em que se procura maximizar a eficiência espectral sem que a probabilidade de erro de bit supere a probabilidade de erro de bit máxima (PEM), cujo valor depende da aplicação [2] e [3]. Esses limiares definem regiões de decisão, cada uma delas vinculada a uma modulação específica. Por exemplo, pode-se empregar a modulação $M_{i}-Q A M$ (em que $M_{i}$ é a quantidade de pontos da constelação da i-ésima modulação) quando $\lambda_{i-1} \leq \gamma<\lambda_{i}$.

Muitos trabalhos divulgados na literatura especializada utilizam esquemas de transmissão adaptativa que assumem que o canal de retorno é livre de erros [4], [5]. Na prática, essa situação pode ser atingida com o uso de poderosos códigos corretores de erro e entrelaçadores com grande profundidade [11]. No entanto, essas estratégias de correção de erro podem adicionar um grande atraso que, por sua vez, podem acarretar degradação de desempenho da técnica de modulação adaptativa. Diante disso, outros trabalhos consideram os erros do canal de retorno e empregam técnicas para contornar ou amenizar a degradação de desempenho da técnica de modulação adaptativa em presença de tais distúrbios.

Os trabalhos [6], [7] modelam o canal de retorno como Canal Binário Simétrico (BSC - do inglês Binary Symmetric Channel). Neles é mostrado que o desempenho do sistema de modulação adaptativa é sensivelmente degradado pelos erros em tal canal, fazendo surgir uma faixa de RSR média no canal avante em que não é atendido o requisito de PEM. Essa região 
é conhecida como região de inviabilidade.

Também em [6], [7] são propostos dois métodos para reduzir o impacto do erro do canal de retorno no desempenho da técnica de modulação adaptativa. $\mathrm{O}$ primeiro admite que o canal avante varia lentamente e impõe restrições quanto à sua evolução entre blocos de dados vizinhos, o segundo baseia-se no emprego de um detector Bayesiano no decisor do canal de retorno.

O trabalho [3] também aborda o problema de erro do canal de retorno, porém nele esse canal é modelado pelo efeito de desvanecimento plano, cuja dinâmica temporal do canal é modelada por um processo Estacionário em Sentido Amplo (ESA) com espectro de Jakes. Além disso, no referido artigo, inclui-se os erros de estimação do canal de retorno na função custo empregada na otimização dos limiares $\lambda_{i}$. Essas considerações permitem vincular a região de inviabilidade a parâmetros típicos de sistemas de transmissão, tais como, o máximo espalhamento Doppler, o tamanho do bloco de dados, a RSR do canal de retorno, sendo mostrado lá que o aumento de qualquer um desses parâmetros amplia a região de inviabilidade. No entanto, no referido trabalho nenhuma estratégia de combate a erros do canal de retorno é avaliada.

Este trabalho avalia o desempenho das estratégias de combate a erros do canal de retorno apresentadas em [6] e [7], porém utilizando para este canal uma modelagem igual à proposta em [3]. Essa modelagem permite elucidar importantes aspectos que não podem ser abordados com a modelagem proposta nos trabalhos [6] e [7].

O restante deste artigo é organizado da seguinte maneira. Uma breve descrição do sistema analisado neste trabalho é dada na Seção II. As duas estratégias para redução do impacto dos erros no canal de retorno são apresentadas sucintamente na Seção III. A Seção IV apresenta os resultados numéricos, enquanto a Seção V apresenta as principais conclusões do trabalho.

\section{DESCRIÇÃo DO SISTEMA}

O diagrama de blocos do sistema de comunicação digital investigado no presente trabalho é apresentado na Fig. 1.

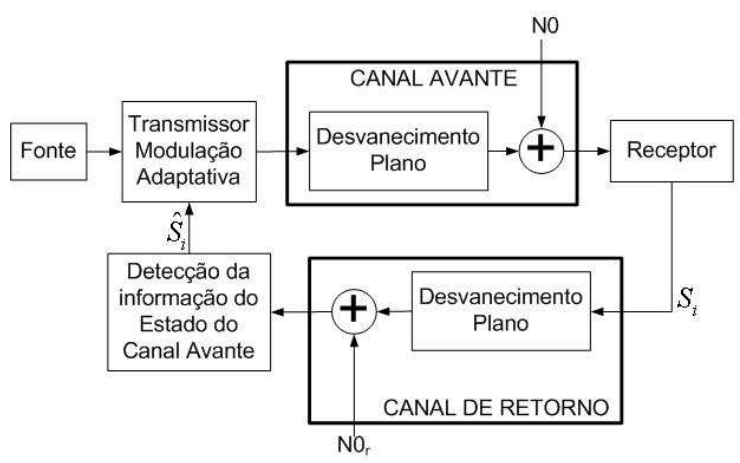

Fig. 1. Diagrama de blocos simplificado do sistema simulado

É assumido que os bits gerados pela fonte são independentes e identicamente distribuídos (iid). Esses bits são entregues ao modulador digital, representado no diagrama pelo bloco Transmissor Modulação Adaptativa, que se encarrega de mapeálos em símbolos $s_{k}$ da constelação da modulação utilizada.
Os símbolos possuem energia média $E_{s}$, independente da modulação adotada.

Neste trabalho, o canal avante e o canal de retorno são caracterizados pelo efeito de desvanecimento plano, cujos ganhos são independentes e modelados por um processo ESA com Densidade Espectral de Potência (DEP) dada pelo espectro de Jakes [8]. Admite-se que o canal de retorno não introduz atraso, diferente do que ocorre em [10], e é estatisticamente independente do canal avante.

O sinal em banda base observado no receptor do canal avante é dado por

$$
y_{k}=h_{k} s_{k}+z_{k}
$$

na qual $h_{k}$ é o coeficiente do canal cujo espalhamento Doppler normalizado é denotado por $f_{D_{A}} T_{A}$, sendo $f_{D_{A}}$ o máximo desvio Doppler e $T_{A}$ a duração do símbolo, ou seja $T_{A}=$ $1 / R_{S}$, em que $R_{S}$ é a taxa de transmissão expressa em baud; $z_{k}$ é o ruído aditivo do canal, modelado por um processo gaussiano branco complexo de média nula e com ambas as componentes real e imaginária independentes e com variância $\frac{N_{0}}{2}$; e $k$ representa o índice do instante de tempo considerado.

O sinal recebido na entrada do transmissor é dado por

$$
y_{k^{\prime}}^{R}=h_{k^{\prime}}^{R} u_{k^{\prime}}+z_{k^{\prime}}^{R}
$$

em que $h_{k^{\prime}}^{R}$ é o coeficiente do canal de retorno, cujo desvio Doppler normalizado é denotado por $f_{D_{R}} T_{R}$, sendo $f_{D_{R}}$ o máximo desvio Doppler do canal de retorno e $T_{R}$ a duração do símbolo; $u_{k^{\prime}}$ é o símbolo transmitido, neste trabalho admite-se a modulação BPSK; $z_{k^{\prime}}^{R}$ é o ruído aditivo do canal de retorno, modelado por um processo gaussiano branco complexo de média nula e com ambas as componentes real e imaginária independentes e com variância $\frac{N_{0}^{R}}{2}$; e $k^{\prime}$ representa o índice do instante de tempo considerado.

Define-se $S_{k}$ como sendo um número natural. $S_{k} \in$ $[0, \ldots, N-1]$, sendo empregadas $\mathrm{N}$ estratégias ou modos de transmissão. $S_{k}$ representa o estado do canal avante durante a transmissão do k-ésimo bloco de dados. Assim, $S_{k}=$ $i$ se $\lambda_{i-1} \leq \gamma<\lambda_{i}$. Nessa notação $\lambda_{0}=0$ e $\lambda_{N} \rightarrow \infty$.

No sistema de modulação adaptativa aqui investigado emprega-se $N$ estratégias ou modos de transmissão. Assim sendo, para cada bloco de dados enviado no canal avante são remetidos $L=\left\lceil\log _{2}(N)\right\rceil$ símbolos para informar ao transmissor o tipo de modulação que deve ser adotada no próximo bloco de dados.

Assumindo que $\mathbf{u}_{j} \in \mathbb{R}^{L}$ denota a sequência BPSK referente ao estado $S_{j}$ e que $\mathbf{y}_{k^{\prime}}^{R} \in \mathbb{C}^{L}$ representa a observação por ela gerada no transmissor, tem-se que

$$
\mathbf{y}_{k^{\prime}}^{R}=h_{k^{\prime} T_{f}}^{R} \mathbf{u}_{k^{\prime}}+\mathbf{z}_{k^{\prime}}^{R}
$$

em que $\mathbf{z}_{k^{\prime}}^{R} \in \mathbb{C}^{L}$ é um vetor aleatório gaussiano cuja matriz de covariância é dada por $\frac{N_{0}^{R}}{2} \mathbf{I}_{L}$, sendo $\mathbf{I}_{L}$ a matriz identidade $L \times L$. Nesta equação admitiu-se que $f_{D_{R}} T_{R} L \ll 1$, de tal modo a tornar razoável a suposição de que o canal de retorno permanece invariante durante a transmissão dos $L$ símbolos, variando entre blocos de acordo com $f_{D_{R}} T_{f}$, em que $T_{f}$ é a duração do frame do canal avante. 


\section{Estratégias AvAliadas}

São propostas em [6] duas técnicas para reduzir o impacto do erro do canal de retorno no desempenho da técnica de modulação adaptativa com o canal de retorno BSC. Tais técnicas são aqui apresentadas de forma sucinta.

\section{A. Detector Bayesiano}

Em sistemas como o tratado no presente trabalho, quando o estado do canal é detectado erroneamente pelo transmissor, duas situações podem ocorrer. Em uma delas o erro provoca a escolha de uma modulação com mais pontos na constelação, produzindo aumento na $\mathrm{EE}$ mas com provável violação da PEM. Na outra, o erro provoca a escolha de uma modulação com menos pontos na constelação, o que ocasiona redução na EE, porém esse erro não provoca violação da PEM. Assim sendo, o primeiro tipo de erro tem consequências mais severas para o sistema de transmissão.

Isso, aliado ao fato de que os estados do canal não são equiprováveis, uma vez que dependem dos limiares $\lambda_{i}$ e da própria modelagem estatística do canal de comunicação, torna apropriado o uso da estratégia de detecção bayesiana para amenizar os efeitos provocados pelos erros no canal de retorno.

Seja $\pi_{j}$ a probabilidade a priori do receptor indicar o esquema de modulação $M_{j}$ como o mais apropriado. Essa probabilidade pode ser expressa por

$$
\pi_{j}=\int_{\lambda_{j-1}}^{\lambda_{j}} f_{\bar{\gamma}}(\gamma) d \gamma=e^{-\lambda_{j-1} / \bar{\gamma}}-e^{-\lambda_{j} / \bar{\gamma}}
$$

em que $f_{\bar{\gamma}}(\gamma)=\frac{1}{\bar{\gamma}} e^{-\frac{\gamma}{\bar{\gamma}}}$ e $\bar{\gamma}=E_{b} / N_{0}$, ( $E_{b}$ é a energia do bit), ou seja, a RSR média do canal avante.

Para a modelagem apresentada na Eq. 3, e admitindo-se o conhecimento do canal de retorno pode-se mostrar que a regra de detecção advinda do emprego do critério bayesiano é dada por

$$
\hat{H}=\min _{i}\left\{\sum_{j=0}^{N-1} C_{i j} \cdot p\left(\mathbf{y}^{R} \mid H_{j}\right) \cdot \pi_{j}, \quad 0 \leq i \leq N-1\right\}
$$

em que $\hat{H}$ é a hipótese mais provável, $C_{i j}$ é o custo associado à escolha da hipótese $H_{i}$ dado que a hipótese correta é $H_{j}, H_{j}$ é a hipótese associada à escolha da modulação $M_{j}, p\left(\mathbf{y}^{R} \mid H_{j}\right)$ é a função densidade de probabilidade da observação $\mathbf{y}^{R}$ dado que a hipótese correta é $H_{j}$, que é dada por

$$
p\left(\mathbf{y}^{R} \mid H_{j}\right)=\frac{1}{\left(\pi N_{0}^{R}\right)^{L / 2}} \cdot \exp \left\{\frac{-1}{N_{0}^{R}}\left|\mathbf{y}_{k^{\prime}}^{R}-h_{k^{\prime}}^{R} \mathbf{u}_{k^{\prime}}\right|^{2}\right\} ;
$$

$\mathrm{Na}$ Eq. 6 a constante que multiplica a exponencial pode ser desconsiderada na avaliação de $\hat{H}$, tendo em vista que tal termo independe da hipótese.

Em razão das discussões apresentadas previamente no que concerne aos efeitos dos erros do canal de retorno no desempenho da técnica de modulação adaptativa, é desejável que os custos presentes na Eq. 5 atendam às seguintes condições: $C_{j j}=0$ para qualquer $j, C_{i j}>C_{j i}$ e $C_{j i}>C_{k j}$ para $i>k>j$. Foi proposta em [7] a seguinte regra para definir os valores dos custos:

$$
C_{i j}=i-j, \quad C_{j i}=\beta C_{i j}, \quad i>j ;
$$

sendo $\beta$ um número real pertencente ao intervalo $(0,1]$.

Com as definições apresentadas na Eq. 7, obtém-se o efeito desejável de se atribuir um custo maior para a escolha de uma modulação com EE maior do que a correta, visando dessa forma reduzir a região de inviabilidade.

\section{B. Detector baseado em Cadeia de Markov}

Nesse método é empregado um detector ML para estimar a sequência BPSK $\mathbf{u}_{j}$ no lado de transmissão, e o canal de retorno é modelado por um Canal Markoviano de Estados Finitos (FSMC - do inglês Finite State Markov Channel). Cada estado corresponde a uma condição de propagação do canal, que é mapeada para uma estratégia de transmissão. É assumido que o canal avante varia lentamente, sendo admitido que no próximo bloco de dados ou ele permanece no estado do bloco anterior ou se encontra em um estado vizinho ao do bloco anterior. Caso a informação recebida não se enquadre nesse padrão, o sistema considera que houve um erro no canal de retorno e o corrige mediante mapeamento do estado detectado para um dos três estados pertinentes, conforme comentado previamente. Assim sendo, assume-se que

$$
P_{i, j} \triangleq \operatorname{Pr}\left(S_{k}=i \mid S_{k-1}=j\right)=0, \quad \text { para }|i-j|>1 ;
$$

na qual e $P_{i, j}$ representa a probabilidade de transição de estados entre blocos adjacentes.

Definindo-se $V_{k}$ como sendo a estimativa ML do estado do canal avante no lado de transmissão para o k-ésimo bloco de dados, e $\hat{S}_{k-1}$ a estimativa do referido estado obtido pelo detector aqui discutido para o $(k-1)$ - ésimo bloco de dados, a estimativa $\hat{S_{k}}$ é obtida da seguinte maneira:

$$
\hat{S}_{k}=\left\{\begin{array}{rll}
\hat{S}_{k-1}+1, & \text { se } \quad V_{k} \geq \hat{S}_{k-1}+1 \\
\hat{S}_{k-1}, & \text { se } \quad V_{k}=\hat{S}_{k-1} \\
\hat{S}_{k-1}-1, & \text { se } \quad V_{k} \leq \hat{S}_{k-1}-1
\end{array}\right.
$$

Esse método é bastante simples, mas possui a desvantagem de ocorrer propagação de erros. Se o estado detectado $\hat{S}_{k}$ não for correto, no próximo instante de tempo a estratégia proposta admite um conjunto de estados possíveis errôneo, baseado em $\hat{S}_{k}$ o que pode afetar as decisões subsequentes.

\section{RESUlTADOS NUMÉRICOS}

Esta seção apresenta resultados numéricos obtidos pelas técnicas de modulação adaptativa que empregam o detector bayesiano e o baseado em Cadeias de Markov para combater os distúrbios provocados pelo canal de retorno, os quais são gerados por meio de simulação de Monte Carlo considerando 8 estratégias ou modos de operação: as modulações BPSK, 4-QAM, 16-QAM, 64-QAM, 256-QAM, 1024-QAM, 4096QAM e a opção de não transmitir.

Para obter cada resultado são transmitidos $10^{8}$ símbolos, compostos da seguinte maneira: cada bloco de dados possui 
10 símbolos, para cada canal sorteado são transmitidos 100 blocos e são sorteados $10^{5}$ canais independentes. A Razão Sinal Ruído média do canal avante $\left(R S R_{A}\right)$ é variada de 0 a $30 \mathrm{~dB}$, com incrementos de $5 \mathrm{~dB}$. Também são simulados vários valores dos produtos do máximo desvio Doppler do canal avante e do canal de retorno pela duração $(\tau)$ do bloco de dados $\left(f_{D_{A}} \tau\right.$ e $\left.f_{D_{R}} \tau\right)$.

Nas situações em que é estimada a resposta ao impulso do canal de retorno é empregado o algoritmo LMS (Least Mean Square), cujo passo foi ajustado para atingir o mínimo valor de erro quadrático médio, conforme resultados analíticos apresentados em [9]. Nas legendas das figuras a serem apresentadas são utilizadas as siglas CRC para canal de retorno conhecido e CRE para canal de retorno estimado.

Na Fig. 2 são apresentadas seis curvas de BER, todas obtidas para uma $P E M=10^{-4}, f_{D_{A}} \tau=10^{-2}$ e $f_{D_{R}} \tau=$ $10^{-2}$, sendo duas obtidas sem o uso de estratégia para redução dos erros do canal de retorno e as demais com o emprego do detector bayesiano proposto em [6] para $\beta=10^{-4}$. Em duas dessas curvas é considerado o erro de estimação do canal de retorno e nas outras esse canal é considerado conhecido. Pode ser visto nesta figura que os erros no canal de retorno provocam o surgimento de uma região de inviabilidade que pode ultrapassar $30 \mathrm{~dB}$ no caso mais crítico $\left(R S R_{R}=10\right.$ $\mathrm{dB})$, quando não há nenhuma estratégia implementada.

Conforme resultados apresentados na Fig. 2, a utilização do detector Bayesiano reduz consideravelmente essa região, chegando até a eliminá-la quando o canal de retorno é conhecido. Em contrapartida, como pode ser visto na Fig. 3, o emprego dessa técnica acarreta uma redução na Eficiência Espectral, sobretudo nas condições mais severas do canal de retorno $\left(R S R_{R}=10 \mathrm{~dB}\right)$, aspecto que não é apontado em [6] e [7]. Cabe ressaltar que mesmo para o caso de canal conhecido essa degradação ocorre.

Resultados semelhantes qualitativamente são obtidos para $P E M=10^{-2}$ e $P E M=10^{-3}$, mas não são aqui apresentados.

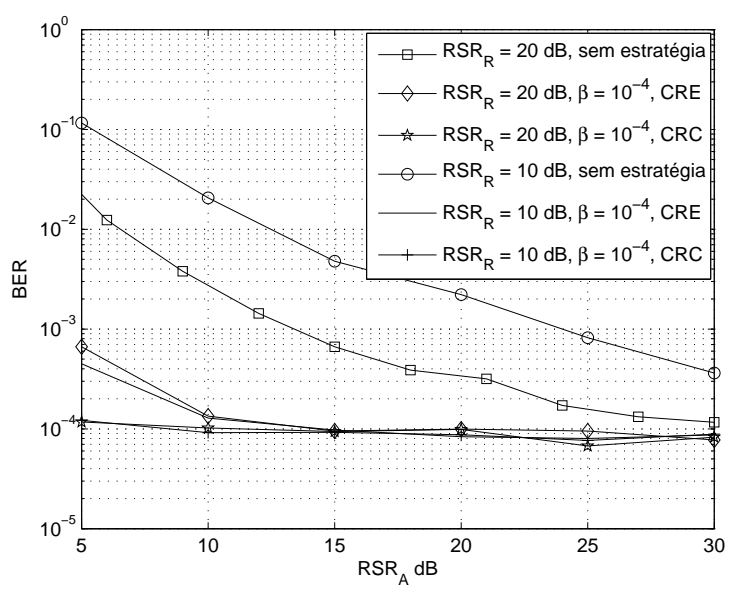

Fig. 2. Taxa de erro de bit com $P E M=10^{-4}$ para $\beta=10^{-4}$.

Para investigar melhor as características de desempenho da estratégia proposta, avalia-se o desempenho da modulação adaptativa em função de $\beta$ para $P E M=10^{-4}$ e $R S R_{A}=$

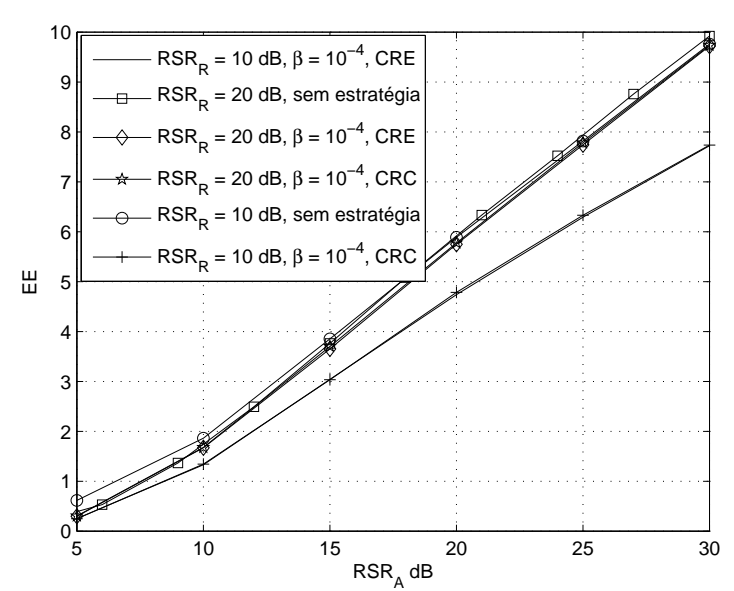

Fig. 3. Eficiência Espectral com $P E M=10^{-4}$ para $\beta=10^{-4}$.

$20 \mathrm{~dB}$, para o canal de retorno conhecido e estimado. Os resultados obtidos são apresentados na Fig. 4. Nessa simulação $f_{D_{A}} \tau$ e $f_{D_{R}} \tau$ são mantidos fixos em $10^{-2}$.

A Fig. 4 mostra curvas de BER em função de $\beta$ para $P E M=10^{-4}$ e $R S R_{A}=20 \mathrm{~dB}$. Nela podemos observar que à medida que se reduz a $R S R_{R}, \mathrm{o} \beta$ deve assumir valores cada vez menores para que seja eliminada a região de inviabilidade. Essa tendência é intensificada quando se passa a considerar o erro de estimação do canal de retorno. Por exemplo, para uma $R S R_{R}=10 \mathrm{~dB}$ com canal de retorno estimado, $\beta \leq 10^{-3}$. Ao passo que para uma $R S R_{R}=30 \mathrm{~dB}$ com canal de retorno conhecido, $\beta$ pode assumir valores próximos de 1 . São encontrados resultados semelhantes qualitativamente para $R S R_{A}=10 \mathrm{~dB}$.

Já a Fig. 5 mostra a Eficiência Espectral obtida sob condições críticas encontradas na Fig. $3\left(R S R_{A}=25 \mathrm{~dB}\right.$ e $\left.R S R_{R}=10 \mathrm{~dB}\right) . f_{D_{A}} \tau$ assume os valores $10^{-3}, 10^{-2} \mathrm{e}$ $5 \times 10^{-2}$, tanto para CRC quanto para CRE. A análise desses resultados indica que a redução de $\beta$ provoca uma degradação da EE.

Essa degradação ocorre mesmo para o caso em que se considera o canal de retorno conhecido, e é mais intensa quando se combinam baixa $R S R_{R}$ e alta $R S R_{A}$. Cabe mencionar que para um mesmo valor de $\beta$ os resultados apresentados na Fig. 5 indicam que a EE é insensível aos erros de estimação do canal de retorno. Porém, de acordo com os resultados apresentados na Fig. 4, para atender a restrição de PEM, em uma mesma condição de $R S R_{R}, R S R_{A}$, e espalhamento Doppler, deve-se adotar um valor de $\beta$ menor quando se considera os erros de estimação do canal de retorno em relação à situação de canal de retorno conhecido. Ou seja, pode-se ajustar o valor de $\beta$ para tentar eliminar a região de inviabilidade, mas se paga por isso uma degradação da EE.

Outro aspecto não tão evidente a partir dos resultados aqui apresentados, mas que foram verificados em diversas simulações realizadas é a dependência da EE com o espalhamento Doppler do canal de retorno quando se leva em conta as estimativas deste canal. Quanto maior esse espalhamento Doppler maior a degradação de BER da técnica de modulação. Assim sendo, menores devem ser os valores de $\beta$ para redu- 
zir a região de inviabilidade, ocasionando assim uma maior degradação da EE.

Assim sendo, $\beta$ exerce um compromisso entre taxa de erro de bit e eficiência espectral da técnica de modulação adaptativa. No sentido que quanto maior $\beta$ menor a degradação da EE em relação à situação de canal de retorno ideal, mas pior a BER. Portanto, o valor de $\beta$ ideal para cada caso é o maior de forma que não haja violação da PEM. Dessa forma, a EE é maximizada com a garantia de cumprimento das exigências quanto à taxa máxima de erro de bits.

Resultados de simulação obtidos, porém aqui não apresentados, indicam que quanto menor a PEM menor deve ser o valor de $\beta$ para que seja eliminada a região de inviabilidade. De forma que para $P E M=10^{-2}$ as degradações verificadas de EE sob as mesmas condições de $R S R_{A}, R S R_{R}$ e espalhamento Doppler, são bem menores do que os aqui apresentados para $P E M=10^{-4}$.

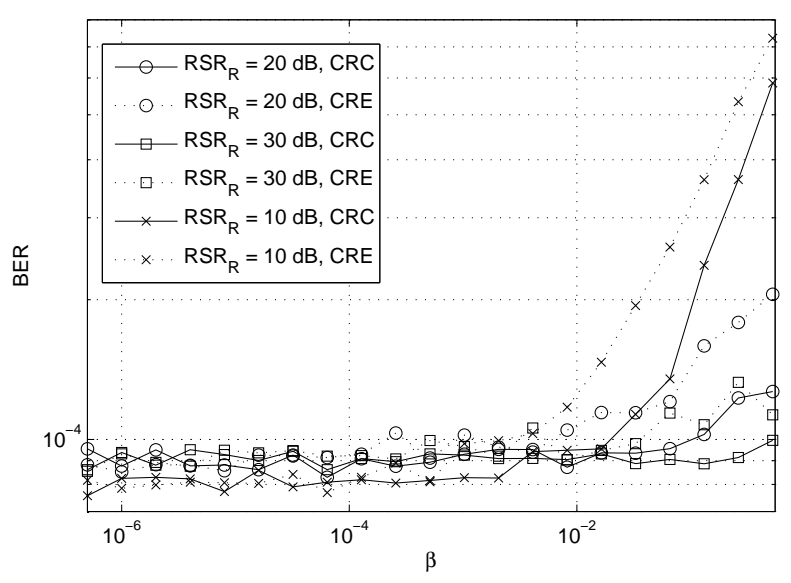

Fig. 4. Taxa de erro de bit com $f_{D_{A}} \tau=10^{-2}, f_{D_{R}} \tau=10^{-2}, P E M=$ $10^{-4}, R S R_{A}=20 \mathrm{~dB}$ em função de $\beta$.

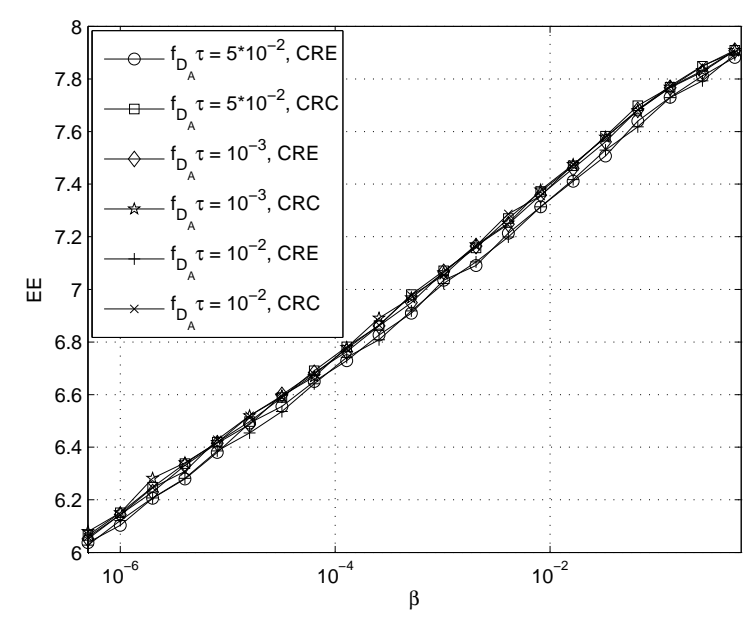

Fig. 5. Eficiência Espectral com $R S R_{A}=25 \mathrm{~dB}, R S R_{R}=10 \mathrm{~dB}$ $f_{D_{R}} \tau=10^{-2}, P E M=10^{-4}$ em função de $\beta$.

A diminuição da EE com a redução de $\beta$, conforme visto na Fig. 3 ocorre em razão da estimativa realizada pelo estimador bayesiano se tornar mais conservadora, pois o custo associado à escolha de uma modulação com menor eficiência do que a que seria utilizada para um canal de retorno ideal é bem menor do que aquela associada à escolha de uma modulação com maior Eficiência Espectral. Cria-se então uma polarização no sentido das modulações com menos pontos na constelação reduzindo a Eficiência Espectral média.

Em suma, a técnica que utiliza um detector bayesiano é eficiente na eliminação da região de inviabilidade, porém apresenta os inconvenientes de degradar a EE em alguns casos críticos, além da necessidade de ajustar o parâmetro $\beta$, cujo valor ótimo depende de vários parâmetros de um sistema de comunicação. Além disso o cálculo de sua métrica requer o conhecimento da variância do ruído. O impacto no desempenho provocado por incertezas com relação a esse parâmetro não são investigados neste trabalho.

Para o detector baseado em cadeias de Markov foram considerados $P E M=10^{-3}, R S R_{R}=20 \mathrm{~dB}, f_{D_{R}} \tau=10^{-5}$ e $f_{D_{A}} \tau$ igual a $10^{-5}, 10^{-4}, 10^{-3}$ e $10^{-2}$.

$\mathrm{Na}$ Fig. 6 são apresentadas seis curvas de BER, todas obtidas para as condições anteriormente citadas, além de se admitir canal de retorno conhecido. Nessa figura foi incluída uma curva para canal de retorno ideal. Pode ser visto também nesta figura que os erros no canal de retorno provocam o surgimento de uma região de inviabilidade que no caso em questão pode ultrapassar $20 \mathrm{~dB}$, quando não há nenhuma estratégia implementada.

Ainda de acordo com a Fig. 6, a técnica apresenta uma boa diminuição na região de inviabilidade para $f_{D_{A}} \tau \leq 10^{-3}$. A região de inviabilidade foi reduzida para $R S R_{A}<9 \mathrm{~dB}$. No entanto, para $f_{D_{A}} \tau=10^{-2}$ a estratégia se mostra ineficaz, obtendo-se um desempenho pior do que sem uso de estratégia. Isso pode ser comprovado com a observação da curva desta figura em que se tem canal de retorno ideal. Mesmo neste caso a técnica introduziu uma grande degradação na BER do sistema, de forma similar aos demais casos, em que o canal de retorno não é ideal, evidenciando, portanto, uma limitação do modelo proposto na Eq. 8. Por outro lado, uma vantagem desta técnica é que não foi verificada redução na EE do sistema. Resultados semelhantes ao apresentado na Figura 6 foram obtidos para $P E M=10^{-4}$ e $P E M=10^{-2}$.

É importante mencionar que $f_{D_{A}} \tau=10^{-2}$ pode ser obtido para um sistema com espalhamento Doppler do canal de retorno muito pequeno mas que apresenta $\tau$ elevado. Por exemplo, em sistemas TDMA, $\tau \geq T_{f}$, sendo $T_{f}$ a duração do frame. Considerando $T_{f}=2 \mathrm{~ms}$, um valor razoável, tem-se que $f_{D_{A}} \tau=10^{-2}$ para um máximo espalhamento Doppler $\left(f_{D}\right)$ de $5 \mathrm{~Hz}$. Isso indica uma séria limitação dessa estratégia de combate aos erros do canal de retorno.

Para investigar melhor as características da referida técnica, é avaliado o seu desempenho em condições mais severas do canal de retorno. $f_{D_{R}} \tau$ assume os seguintes valores: $10^{-4}$, $10^{-3}$ e $10^{-2}$. Considerou-se $R S R_{R}=10 \mathrm{~dB}$ e canal de retorno estimado.

A Fig. 7 apresenta seis curvas de BER. Duas curvas com canal de retorno conhecido são empregadas como referência: uma delas considerando $f_{D_{R}} \tau=10^{-5}$ e $R S R_{R}=20 \mathrm{~dB}$, e outra com $f_{D_{R}} \tau=10^{-2}$ e $R S R_{R}=10 \mathrm{~dB}$. As outras curvas 
consideram canal de retorno estimado. É possível perceber que conforme as condições de propagação do canal de retorno se tornam mais severas, a região de inviabilidade se intensifica.

Em suma, a técnica não é satisfatória em cenários em que o canal de retorno apresenta condições ruins de propagação (baixos valores de $R S R_{R}$, altos valores de $f_{D_{R}} \tau$ e canal de retorno estimado), bem como para canais avante com moderados valores de $f_{D_{A}} \tau$.

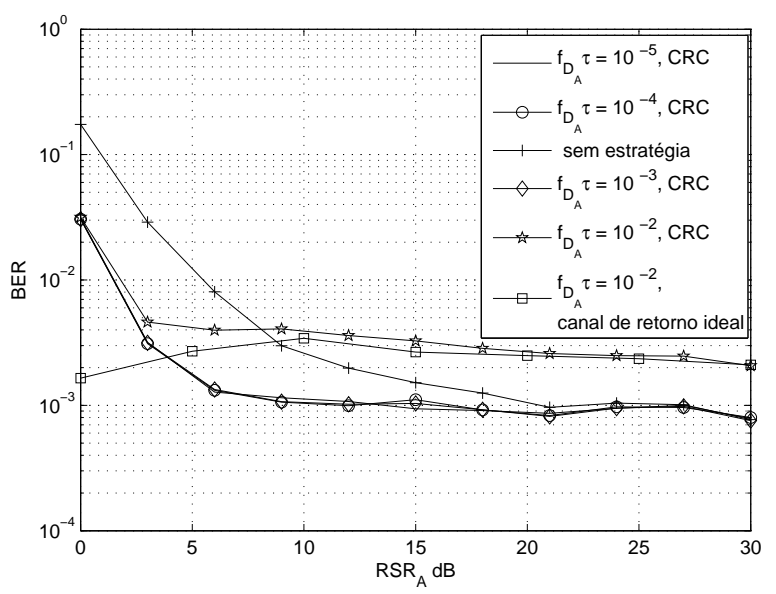

Fig. 6. Taxa de erro de bit com $P E M=10^{-3}, f_{D_{R}} \tau=10^{-5}$ e $R S R_{R}=$ $20 \mathrm{~dB}$

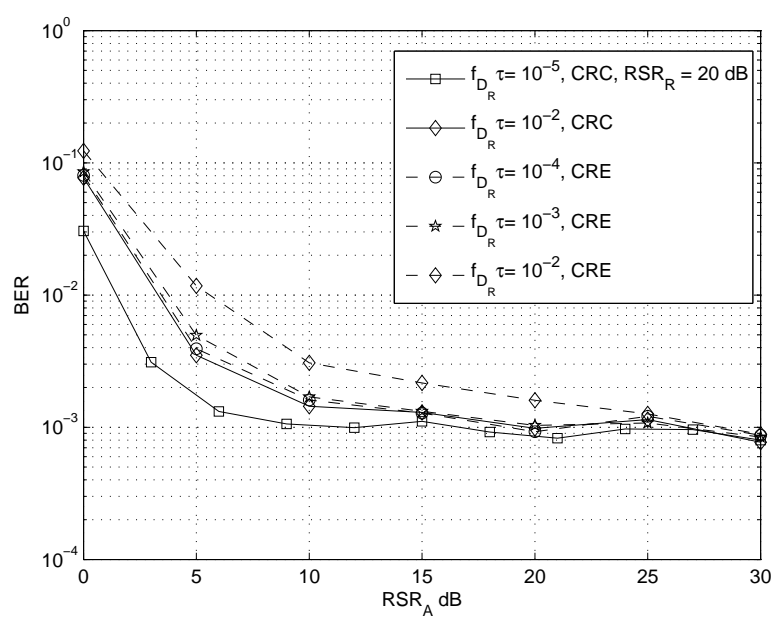

Fig. 7. Taxa de erro de bit com $P E M=10^{-3}, f_{D_{A}} \tau=10^{-4}$ e $R S R_{R}=$ $10 \mathrm{~dB}$

\section{CONCLUSÕES}

Neste trabalho realiza-se a avaliação de desempenho de técnicas de modulação adaptativa que empregam as estratégias propostas em [6] para reduzir a região de inviabilidade (o detector bayesiano e o detector baseado em cadeias de Markov), que caracteriza tais técnicas quando empregadas em canais de retorno que apresentam erros. No entanto, a avaliação aqui realizada emprega um modelo de canal de retorno diferente do adotado no trabalho [6], e que apresenta algumas vantagens em relação à modelagem original, pois permite estabelecer cenários apropriados para o emprego das estratégias investigadas. Além disso, outra contribuição deste trabalho é a avaliação de desempenho dessas técnicas quando ocorre erro de estimação do canal de retorno, assunto não abordado em [6].

Conforme mostrado nas seções anteriores, as técnicas simuladas propiciam boa melhora de desempenho, tendo diminuído ou até eliminado a região de inviabilidade do sistema. A estratégia bayesiana apresenta resultados superiores ao da técnica baseada em Cadeias de Markov pois é capaz de eliminar completamente esta região, mesmo quando o canal de retorno é estimado, desde que se escolha um valor de $\beta$ baixo o suficiente. Em contrapartida a técnica bayesiana degrada a eficiência espectral, sobretudo quando o canal de retorno é estimado. Para reduzir essa degradação é necessária a escolha de um valor de $\beta$ para cada conjunto de parâmetros de um sistema de comunicação, o que é outro inconveniente desta técnica.

Já a estratégia baseada em Cadeias de Markov apresenta a vantagem de não afetar a EE e de nem requerer o ajuste de parâmetros, mas apresenta duas limitações sérias: a incapacidade de eliminar completamente a região de inviabilidade e a severa restrição do modelo adotado para o canal avante, de tal modo que o seu emprego é inadequado para canais avante com $f_{D_{R}} \tau \geq 10^{-3}$.

\section{AgRADECIMENTOS}

Os autores desejam agradecer o apoio financeiro prestado pelo Conselho Nacional de Desenvolvimento Científico e Tecnológico - CNPq - Brasil ao desenvolvimento deste trabalho (Edital MCT/CNPq 15/2007 - Universal), bem como as valiosas sugestões dos revisores anônimos.

\section{REFERÊNCIAS}

[1] PROAKIS, J. G. Digital Communications. McGraw-Hill, 4a Ed., 2000.

[2] SVENSSON, A. An Introduction to Adaptive QAM Modulation Schemes for Known and Predicted Channels. Proceedings of the IEEE, Vol. 95, No. 12, pp 2322-2336, Dezembro 2007.

[3] GALDINO, J. F., GURJÃO E.C., Otimização de Limiares para Adaptação de Modulação Diante de Erros no Canal de Retorno. XXVI SBrT, Rio de Janeiro, Setembro 2008.

[4] TORRANCE, J.M. e HANZO, L. Optimization of switching levels for adaptive modulation in slow Rayleigh fading, IET Eletronics Letters, 32(13):1167-1169, Junho 1996.

[5] TORRANCE, J.M. e HANZO, L. Upper bound performance of adaptive modulation in a slow Rayleigh fading channel, IET Eletronics Letters, 32(32):718-719, Abril 1996.

[6] EKPENYONG, A E. and HUANG Y-F. Feedback-Detection Strategies for Adaptive Modulation Systems. IEEE Transactions on Communications, Vol. 54, No 10, pp 1735-1740, Outubro 2006.

[7] EKPENYONG, A E. and HUANG Y-F. Feedback Constraints for Adaptive Transmission. IEEE Signal Processing Magazine, pp 69-78, maio 2007.

[8] PARSONS, J.D., The Mobile Radio Channel, John Wiley, 1992.

[9] GALDINO, J.F., PINTO, E.L., ALENCAR, M.S., Analytical Performance of the LMS Algorithm on the Estimation of Wide Sense Stationary Channels, IEEE Transactions on Communications, Vol 52, No 6, 2004.

[10] GOECKEL, D. L., Adaptive Coding for Time-Varying Channels Using Outdated Fading Estimates, IEEE Transactions on Communications, vol 47, No 6, pp 844-855, junho 1999.

[11] LEUNG, C., KWAN, R., Adaptive Modulation and Coding with Multicodes over Nakagami Fading Channels, IEEE Wireless Communications and Networking Conference, New Orleans, Março 2005. 